\title{
About the new industrial production management concept as the company strategy in the fourth industrial revolution
}

\author{
Julia Kovalchuk $^{1, *}$, and Igor Stepnov ${ }^{2}$ \\ ${ }^{1}$ Market Economy Institute of Russian Academy of Sciences, Moscow, Russia \\ ${ }^{2}$ Ryazan State Radio Engineering University, Ryazan, Russia
}

\begin{abstract}
The new industrial production management requires a review of the third industrial revolution results and accounting for mass adoption of information and communication technologies to create the organizational basis of the fourth industrial revolution. The future changes will affect all components of the organization and management components of industrial enterprises, forming the potential of new competitive advantages in a global economy. The research included the identification of key factors of formation, development and destruction (absorption related branches of knowledge) the industrial production management as the theory and practical activities, given the critical approach to its nature and processes. Revealed common signs of the industrial production management need as a field of knowledge in the framework of previous and current industrial revolutions. It is shown that the industrial production management effectively solves the problem of subsistence economy, and substantiates that the modern digital economy also has the characteristics of subsistence economy. It is important the necessity of formulation of a new organizational thinking, the implementation of which is possible in the modern interpretation of the project office. The article represents the theoretical basis for developing practical recommendations for the formation of the new concept of industrial production management to take advantage of the impact of engineering component on the economic results and the creation of project offices for the development of traditional and created markets in the organization of a new production mode (based on the digital economy).
\end{abstract}

\section{Introduction}

The phenomenon of the industrial production management that prevailed in management science, education and practice in the last century in Russia resulted in almost complete disappearance as such of the educational program subject (the study discipline) that once required for all engineers and organizers of production, the absence of a recharge new scientific research, and, in general, lack of popularity among practitioners.

The importance of the industrial production management for the domestic industry was due to the fact that it was the locomotive to unite and lead the industry towards efficiency of technical and technological renewal of industry.

What has caused the role of the industrial production management in organizational and managerial sphere? Why today, the industrial production management is fragmented into a number of components and, in many ways, absorbed by the other branches of knowledge? What are the future prospects of this direction? Here are the key questions which must be answered to researchers in this field of knowledge, putting the fundamental question about the nature of organizational and managerial solutions at the present time.

\section{Relevance}

A new round of technological progress, which has given a significant number of hypotheses about postcapitalism [1], largely changed the economic model of society. Therefore, in our opinion, the strategic principles and approaches laid down several decades ago to the creation of an area of knowledge as the industrial production management, should be used for the formation of the new industrial production management. In fact, we must recognize that new technology:

i. change the relationship between labor and wages (just as a century ago mass production made the same discrepancy);

ii. destroy the action of the "invisible hand of the market", disrupting the market pricing (just as mass production has fundamentally changed the pricing of commodities);

iii. allow to create a new integration in joint production (similar to the formation of corporations a century ago). 
Despite the fact that the concept of the third industrial revolution (based on the introduction of new energy sources in conjunction with information and communication technologies [2]) is not fully formed (and the revolution has not ended because of the speed and the magnitude of the current changes), there are some concepts of the next (the fourth) industrial revolution:

- Industrie 4.0 [3] is the creation of digital enterprises based on the digitalization of all systems (physical assets) and their integration into the digital ecosystem with the partners involved in the value chain;

- the new industrial revolution of the "makers" by K. Anderson [4] is the creation of customized mass production with the possibility of interactive exchange of ideas and developments based on the development of 3D design and 3D printing and use the additive technologies;

- the fourth industrial revolution by K. Schwab [5] is the transition from the simple digitalization (as the third industrial revolution) to the innovations, based on combinations of technologies (the fourth revolution), to information platforms, combining the supply and demand and in violation of existing production structures, the creation of new organizational forms and business models like "sharing" economy and "ondemand" economy;

- the new (the fifth) industrial revolution by P. Marsh [6] is a new industrialization of the economy in developed rich countries, which will affect the worldwide industry.

Despite the significant number of opinion and of the terms that generated by the revolutionary transformations in an increasingly technological society, economy and business management should distance themselves from the trappings of change and must focus on finding a compatible principles, approaches and concepts, instead of declarative discussing areas.

The industrial production management in the classical sense is essentially "subsistence economy" [7], and sees "problems" as "reserves" for future development [7], that substantially distinguishes the industrial production management, for example, from the controlling concept [8]. As evidence, it should be noted that many of the actions in the information world have become a kind of subsistence economy, but only informational - the same video or book piracy in its essence is a natural exchange of information when creating a new (libraries, Wikipedia, etc.).

\section{Discussion and theoretical justification}

\section{Formation, development and destruction of the industrial production management key factors}

Later in this article, we examine the factors that influence the formation, development and destruction of the industrial production management.

\subsection{Placement and time}

At first back to basics and define what the industrial production management solved the problems of interaction in placement and time as the key scarce resource, which had a maximum limit (placement due to high cost of the production area, and time as factor productivity).

Point of view on the concept of " the industrial production management" was formed from mid 30's to the 70's of the last century. Among the most well-known scientists, whose works have formed this base of knowledge should include the following scientists: B.Ya. Kachenbogen, L.Ya. Shukhalter, S.A. Heinman, E.A. Satel, S.E. Kamenitzer, A.I. Neimark, I.M. Razumov, N.A.Slodkevich, V.A. Letenko etc. [7, 9-20].

The industrial production management is presented as the combination and distribution in placement and time personal and physical factors of production with the specific technical conditions of the production. However, later, depending on the prevailing economic conditions, environmental effects, or lack thereof influence, the interpretation of the notion " industrial production management" looked different, refined and specified from the perspective of systems theory and applied aspect for almost all sectors of the economy. The most significant difference between the developed in those years methods is their simplicity, obviousness, the possibility of a recurrence and guarantee a stable result.

A special place in the industrial production management took operational production scheduling, which subsequently spawned a new branch of knowledge, but that operational production scheduling has largely determined the growth of labor productivity in the 60's, reduce lost production time [16].

\subsection{Scientific and technical progress}

Without a doubt scientific and technical progress had a huge impact on the production processes, in particular it requires a comprehensive solution of the issues of products modernization and the creation of new and development of their production technology and production management. It should be noted that most of the organizers of production, solving the enterprise's problem of organizing placement and time for a short time interval, when considering the issue of productivity growth went to the mandatory consideration of the influence of scientific and technical progress [7, 13]. The development of market economy in Russia caused a change in requirements for industrial production management, which came to be regarded as a particular environment in which the conditions for the best use of people and technology in the production process, thereby increasing its effectiveness [21].

On the forefront there are the problems of competitive products production in the shortest possible time, reducing the serial and batch size, improve the quality in accordance with world standards, skills development and creative potential of employees in terms of improving equipment and technology, improving the flexibility and mobility of production. 
In accordance with the changed approaches to determining the industrial production management. So, in [17] define the essence of industrial production management in the integration and the interoperability of personal and physical elements of production, the establishment of necessary links and coordinated actions of participants of production process, creation of organizational conditions for realization of economic interests and social needs of workers in the industrial enterprises.

Thus, in addition to orientation to key resources (which were scarce and crucial not only in the market economy, but also in the planned economy), industrial production management became the integrator of all production processes, flexibly responding not only on industry characteristics but also on the challenges of our time such as technological progress and automation. And for many generations of Industrialists, the industrial production management became a symbol of renewal, a symbol of progress (especially in terms of mass production). Modern development of production infrastructure and a significant increase in the productivity of the equipment (not labor!) led to the reduction of the role of these factors in production.

\subsection{Modularity and the possibility of a recurrence}

The big advantage of the production was the possibility of organizational solutions recurrence, which guaranteed a stable result. Such a recurrence was possible not only in one industry, but is applicable for various industries (i.e. the production type was more dominant symptom than industry characteristics). One of the directions of development of organizational science was the search for modular integrated solutions. Among them should include the organizational platform [22], when the decisions on the industrial production management was added to the institutional aspect. But, as shown by the development of the economy over the last fifteen years, the organizational platform confirm their objective existence at a level higher than the enterprise, and practically are not in demand at the enterprise level. The organizational platform is inherently designed to limit the market variety of forms of interaction (similarly to the emergence of neo-institutional theory, when the institutional theory was not able to fully replace the monetary theory, then there is an institutional attachment to the main theory). And thus, the organizational platform as a modular design that reinforces the relationship in the market environment, were in demand only at the level of the enterprises and above.

\section{A. The Productivity and Mass Production}

The universality of the industrial production management in its golden age was confirmed by the fact that regardless of industry and size of the enterprise, its principles assumed productivity growth and has become an essential element of development.

Mass production was dictated by the demand (market or plan), and it should be noted in the period a large number of research devoted to the increase of serial (from order to small-scale, from small-scale to largescale and until the mass, as the ideal is mass production). The main impact on this concept's component was the principles of flexibility as a response to market needs and manufacturing automatization [23].

The victory of the new industrial revolution will be achieved only if the production in the digital economy will provide a commensurate in the early stages the level of costs and productivity in mass production. Still, such a commensurability could be observed at the level of replacement of structures and materials (e.g., replacement of steel hull to aluminum polycarbonate, etc.). Development of 3D printing technology can form a distributed network as open to the consumers and does not require skilled workers. This approach to the fourth industrial revolution, more recognized in Germany, could lead to mass de-industrialization large-scale production.

\subsection{System approach}

Traditionally a strong point of the industrial production management is the proclamation and adherence of a system approach. But, paradoxically, a system approach also made a significant contribution to the reduction of the role of the industrial production management in its attempt to spread the industrial production management in all spheres of industrial knowledge. It is an attempt of generalization and universality led to erosion and separate the industrial production management's thesis to the other fields of knowledge and destroyed the science by the reason of formalization and generalization of knowledge instead of protecting the target segment and increase knowledge about the object of the industrial production management.

Instead of development of the production engineering methods and interaction within the enterprise the industrial production management began to explore all aspects of the enterprise (from industrial engineering to consumer services) that as a result led to the organizational principles began to be developed and enhanced, but the generalization has led to the emergence of the laws of the industrial production management, but did not add new meaning.

\subsection{Engineering solution and the cost}

The following strong component of the industrial production management was the connection of engineering and efficiency, in which engineers developed the solutions, the justification for which was based on the product cost. This result was achieved without depending on financial and economic departments because the cost was the engineering option[9, 12-14].

This component of the industrial production management was also reformed in the time of arrival of financial managers in companies management. This was achieved due to the replacement of the cost by the budgets and production budgets in the basis of 
management. Then production managers instead of cost reduction, productivity growth and technical updates have become the busy budgeting. The result is another group of costs, which have led to different results, because the manipulation of figures is more effective for obtaining the financial result, but less useful from the point of view of productivity growth.

Understanding the importance of budget planning and the need to value thinking for every managing at any level, should still be noted that financial science is very well appreciated experience of the industrial production management knowledge and actively replaced it with business processes organization.

On the one hand, this process has been objective in mind of reducing the requirement for mass production and the growth performance of the equipment (i.e. reduce the relevance of issues), but on the other hand, it was initiated by financial department of the companies to strengthen their position in the hierarchy of the company's management. It led to significant contradictions between the financial and operational management with a victory of financial management [24].

\subsection{Advance}

An important stage of development of the industrial production management was an attempt to make advance nature of production, i.e. attempts to create the industrial production management to meet the challenges of the time: a mechanism of ensuring competitive advantages should be based not only on responding to a dynamic and every time a qualitatively new changes in the external environment, and the pre-emption by changing their own behavior. As a result of changes in the market and offset customers needs has transformed the production concept: focus on efficiency it has passed to focus on quality, flexibility, innovation and, ultimately, to reduce development time for new products.

Therefore, with respect to the industrial production management necessitated orientation of its proactive as a property caused by the dynamic external environment and the requirement of competitiveness of an industrial enterprise and its products. Moreover, the advance is treated in precisely temporally, because, considering the industrial production management as a combination of elements of the production process in time and in placement, ahead in placement, it is objectively impossible [25].

The advance of the industrial production management can be regarded as strategic and tactical. Strategically advance of the industrial production management aimed at accelerating the achievement of the competitiveness of the production system. The advance is determined by the ability to make rapid changes in development and production of new products, anticipating changes in the external environment, in particular, emerging needs of consumers and the corresponding market conditions.

While tactically advance can be seen as directly based on the adaptation of the industrial production management to changing conditions of functioning of the production system based on innovation of industrial production management. The advance and the adaptation are not opposed, but complementary.

Therefore, the proactive industrial production management is seen as such the industrial production management that has the ability to provide rapid changes in the processes of new products setting to the production and to establish its accelerated release by the deadline in accordance with the market requirements.

\subsection{Criteria of management efficiency}

Another important characteristic of the industrial production management was a rejection of the techniques and methods of management at the production processes level (or rather, their inclusion in the industrial production management, thus ensuring automatic compliance). Proper industrial production management ensures the automatic generation of activity indicators depending on the production method, and many of the issues addressed by the labor regulation, not a set of balanced indicators.

This advantage was also deformed initially by management as a versatile tool (instead of the balance between technical and managerial solutions), and then financial departments. Financial management here won the industrial production management, introducing a system of indicators which must be monitored with a high degree of abstraction, again forgetting the principle that well-organized production system contained specific indicators within the system and not demanded the introduction of a new one.

As an example should bring the cost of information and communication technologies, showing that manufacturers have long understood the importance and value of the cost information, however, financial experts are still not bothered about the introduction of a new element to the cost of information and communication technologies [24, 26].

\subsection{Project approach}

The industrial production management always had the project approach and was the part of preproduction. The ideas and methods of the industrial production management began to be actively used in project management, but project management methodology did not consider it necessary to delve into the detail of the projects, leaving this part of the work of technical professionals, and focused on planning and reporting.

In particular, it should be noted that activity network and system PERT contained (including the content and duration) and the outcomes of actions. However, modern technologies operate on information reports about the completion of actions, which in turn also alienated the projects management from the industrial production management.

It should be noted that for project management the subject of exchange is information, but for production the subject of the exchange is product. Thus, it is 
possible to consider project management a kind of derivatives products, which leads to relevant risks despite the fact that brings natural economy to commodity production.

\subsection{The Communication protocol}

The industrial production management have always created a communication protocol, which is independent from the contractor (and the industrial production management even has introduced the forced nature of such interaction) and the most ignored cognitive distortions. These decisions have resulted in subsequent generations organizational solutions were already implemented in the framework of the new project and the need for the industrial production management decreased. Changing organizational reality leads to the fact that many of the standard methods no longer provide the required quality, and in the new organizational reality there was the possibility of multiplicity of solutions. Currently, it should be noted that the increasing role of cognitive distortions and the relevant risk became prevalent in the production planning, and addressing them is one of directions of development of modern industrial production management [27].

In addition, should talk about the cognitive distortions that can appear at the information and robotic systems not only in their interaction with man, but also in their interaction between themselves because the protocols, written by different programmers, can allow such opportunities, which were not originally planned. Also, if we assume that these devices are also studying the world and while not dangerous in its activities, but in the near future, the protocols of exchange between them will be a significant problem, since we can't even imagine what kinds of interaction and organization calculates by the machine intelligence. Therefore, the new industrial production management should consider communication not only workers, but also information and robotic systems.

\section{The decisions and practical significance}

\section{The Prospects of a new organizational reality}

So, the strength of the emerging industrial production management was engineering and the production cost, which gave the maximum result. The need for further productivity growth has led to the fact that the industrial production management began to distribute their solutions in the design stage and the effects of scientific and technological progress. But in the countries with market economy (unlike Russia) the expansion of operational management was aimed at marketing sector, with the aim of reducing costs.

Today engineering is largely starting to be replaced by startup, which create a wide range of solutions with the possibility of selection. This phenomenon is due to the fact that the majority of engineering solutions transformed from the only allowable in the possible, because the task of consumption or provide supply in a particular segment have been solved, and the vast majority of engineering decisions are in the improvements nature, not revolutionary changes. On the one hand, it is indicates that is no urgent need for these decisions to society, and the industrial production management in the old sense not in demand. On the other hand, preserved the industries, for which engineering has maintained its significance (e.g., energy).

On this basis, the new industrial production management may be presented in the two strategies form:

i. the strategy for those markets that are turning scarce resources and which are mandatory for human life - for example, food, energy, etc. In this area it is maximum necessary to develop standard processes (including, in addition to the foregoing, and quality assurance). It is very common for these industries is a preference for the formation of a system of indicators. But the system of indicators there is one danger - it needs to constantly innovate and prove the usefulness of these indicators. Hence, for modern managers is important to understand that the system of indicators, like the budget system, may lead to manipulation of data, not efficiency. In addition, the system of indicators, like any accounting system, works well with already a fait fact, did not react to expected changes, and may become a factor of destabilization. The industrial production management reduces the future risks (contributing to the dilemma of risk-return), just reducing the risk at the expense of repeatability, stability and higher productivity, giving opportunities for risk taking. A key task of the industrial production management is its upgrading to ensure readiness for the adoption of the fourth industrial revolution;

ii. strategy for unformed markets, on which startups start their activity, and thus future reality design is key for them. For those markets in which demand is determined by marketing, fashion, desire updates, etc., the digital economy can begin to destroy the market pricing, being focused on the market design with the specified parameters. It is therefore very important to focus on organizational training to the new market, not preproduction.

As for marketing [28], here one should set an analogy with the cost. If early mandatory for the success was the unification of engineering and cost control, today for a startup case, in addition to the cost should be included the marketing results. Of course, the industrial production management should not be totally absorb marketing. Marketing should be the cornerstone of market design, i.e. to precisely define the object of exchange in the designed system, in order to proceed with the market design. It should be noted that the organizers of the production have missed there chance in Silicon valley, which the experience was to give a new 
impulse to the organizers of the production. However, focusing at the moment the other segment (for mass production), they are not considered signals of new time a significant challenge.

\section{Conclusion}

As paradoxical as it sounds, for the revival of the industrial production management need a cognitive approach, information support and marketing included in the knowledge conductor of the organizational decisions. The Western world has chosen today startup as a conductor instead of engineer, creating a whole sphere of the knowledge economy and including startup to the large corporations activities. But this does not mean that the general orientation to the project approach will create new industries, skipping the stage of the industrial production management. The effectiveness of the projects will not increase by speculation the word "project", and the design thinking can lead to the departure from idea to technology implementation ideas - this approach has never been progressive at a strategically important time. Therefore, we propose to expand the functional framework of the project office, re-establishing the principles of the industrial production management in the formation of a new mode of production (based on the digital economy) and ensuring effective interaction design approach with cognitive technologies, information and design market.

These technologies should be a source of renewal of the industrial production management, but not budgeting, not the Balanced Scorecard, not project management, which are not productive in the early stages of creating a new market. For such tasks, it is necessary to create a project office based on the new organizational reality. The project office, which is based on the real product of the exchange, should ensure the connection all major elements of the new digital economy.

The authors would like to thank the Russian Science Foundation for the financial support in the realization of the research project No. 16-18-10149. The authors are also grateful to the Market Economy Institute of Russian Academy of Sciences for making scientific work.

\section{References}

1. P. Mason PostCapitalism: A Guide to Our Future (AdMarginem, Moscow, 2016)

2. J. Rifkin, The Third Industrial Revolution: How Lateral Power Is Transforming Energy, the Economy, and the World Hardcover (Alpina nonfiction, Moscow, 2014)

3. Industry 4.0: Building the digital enterprise. http://www.pwc.ru/en/technology/publications/in dustry-4.html.

4. C. Anderson Makers. New industrial revolution (Gardners Books, 2011)
5. P. Marsh, The New Industrial Revolution: Consumers, Globalization and the End of Mass Production (Yale University Press, 2013)

6. K. Schwab, The Fourth Industrial Revolution (The publishing house E, Moscow, 2017)

7. S.A. Heinman, Industrial production management at the USA enterprices (The foreign literature publishing house, Moscow, 1961)

8. J.K. Liker, The Toyota Way: 14 Management Principles From the World's Greatest Manufacturer (cGraw-Hill, Moscow, 2003)

9. B.Ya. Katsenbogen, The Industrial Production Management in Machine Building (The Central scientific-research Institute of industrial organization and management, Moscow, 1937)

10. L.Ya. Shukhalter, The Industrial Production Management in Machine Building (USSR Industrial Academy, Moscow, 1938)

11. L.Ya. Shuhgalter, Yu.S. Veniaminov, E.K. Smirnitsky, Economics, Formation, Planing, Management (Economika, Moscow, 1985)

12. E.A. Satel, Formation, Planing and Economics of the Producing Department Machine-Building Plants (Mashgiz, Moscow, 1962)

13. S.E. Kamenitzer, The Industrial Production Planning and Management (Politizdat, Moscow, 1967)

14. A.G. Berman, A.I. Neimark, Production Line Methods in the Serial Machine Building and Instrument Making (Mashgiz, Moscow, Leningrad, 1958)

15. I.M. Razumov, L.A. Glagoleva, M.I. Ipatov, V.E. Ermilov, Formation, Planing and Management in Machine Building Plants (Machine Building, Moscow, 1982)

16. N.I. Slodkevich, N.A. Salomatin, N.V. Andreeva, Operative Accounting and scheduling at the Machine-Building plant (MIYu, Moscow, 1980)

17. V.A. Letenko, O.G. Turovetch, The MachineBuilding Industrial Production Management: Theory and practice (Machine Building, Moscow, 1982)

18. V.I. Tikhomirov The Production Management (MAI, Moscow, 1986)

19. I.M. Razumov, Production Line and Serial-Scale Production Formation in Machine Building (The state Committee of the USSR Council of Ministers for the coordination of scientific research, Academy of Sciences of the USSR, Moscow, 1961)

20. V.F. Ershov, N.P. Smirnof, O.A. Yumina, Industrial The Production Management at the Department of the Machine Building Plant in the market conditions (University of engineering and Economics, Saint-Petersburg, 2004) 
21. R.L. Satanovsky, Organizational Flexibility of the Machine-Building Production (Machine Building, Leningrad, 1987)

22. I. Stepnov, The Theory and methodology of the Innovative Potential Usage in the Regional Industry (Saint-Petersburg, 2001)

23. I.M. Stepnov, T.G. Sadovskaya, Mashinostroitel, 8 (1990)

24. I. Stepnov, Economics and management in mechanical engineering, 6 (2014)
25. J. Kovalchuk, Organizer of production, 1, 24 (2005)

26. J. Kovalchuk, I. Stepnov, Proceedings of The International Conference, Management and controlling in the conditions of instability of markets and external threats (2015)

27. A. Kazelskaya, I. Stepnov, Modern economics: problems and solutions, 5 (2016)

28. V.V. Glukhov The Industrial Production Management and Marketing (Polytechnical University, St. Petersburg, 2012) 